\title{
Isotopic production cross sections of residual nuclei in the spallation reaction ${ }^{136} \mathrm{Xe}(200 \mathrm{~A} \mathrm{MeV})+p$
}

\author{
C. Paradela, ${ }^{1,2,{ }^{*}}$ L. Tassan-Got,${ }^{1}$ J. Benlliure,${ }^{2}$ J. L. Rodríguez-Sánchez ${ }^{2}$ L. Audouin, ${ }^{1}$ A. Boudard,${ }^{3}$ E. Casarejos,${ }^{2, \dagger}$ \\ T. Enqvist, ${ }^{4}$ J. E. Ducret, ${ }^{3}$ F. Farget, ${ }^{1,+}$ B. Fernández-Domínguez, ${ }^{3,}{ }^{\S}$ M. Fernández Ordóñez, ${ }^{2}$ L. Giot, ${ }^{4, \|}$ A. Heinz, ${ }^{4, \pi}$ V. Henzl, ${ }^{4}$ \\ D. Henzlova, ${ }^{4}$ A. Kelić-Heil, ${ }^{4}$ A. Lafriaskh, ${ }^{1}$ S. Leray, ${ }^{3}$ P. Napolitani, ${ }^{1}$ J. Pereira, ${ }^{2, \#}$ D. Pérez-Loureiro, ${ }^{2, \#}$ M. V. Ricciardi, ${ }^{4}$ \\ C. Stéphan, ${ }^{1}$ K.-H. Schmidt, ${ }^{4}$ C. Schmitt, ${ }^{4, \ddagger}$ C. Villagrasa,${ }^{3}$ C. Volant,${ }^{3}$ and O. Yordanov ${ }^{4, * *}$ \\ ${ }^{1} I P N$, CNRS/IN2P3, Université Paris-Sud 11, F-91406 Orsay, France \\ ${ }^{2}$ Universidad de Santiago de Compostela, E-15782 Santiago de Compostela, Spain \\ ${ }^{3} I R F U, C E A$, Université Paris-Saclay, F-91191 Gif-sur-Yvette, France \\ ${ }^{4}$ GSI-Helmholtzzentrum für Schwerionenforschung GmbH, D-64291 Darmstadt, Germany
}

(Received 31 January 2017; published 10 April 2017)

\begin{abstract}
The residual nuclei produced in the spallation reaction of ${ }^{136} \mathrm{Xe}$ nuclei at $200 \mathrm{~A} \mathrm{MeV}$ on protons have been studied by measuring the isotopic distributions for the elements from cadmium $(Z=48)$ to cesium $(Z=55)$ by using the fragment separator (FRS) spectrometer at GSI and the inverse kinematics technique. This is one of the few measurements performed at such a low projectile energy, close to the validity limit for intranuclear cascade models such as INCL or ISABEL. The experimental results have been compared to these intranuclear cascade codes coupled to the evaporation code ABLA. Both code combinations reproduce qualitatively the measured isotopic distributions; however, both underestimate the production of residues with mass numbers between 126 and 134 . The measured cross sections are of interest for the planning of future radioactive beam or neutron source facilities.
\end{abstract}

DOI: 10.1103/PhysRevC.95.044606

\section{INTRODUCTION}

Spallation reactions refer to nuclear reactions between an energetic light particle, usually a proton, and a heavy nucleus, with the projectile energy being between $150 \mathrm{MeV}$ and several $\mathrm{GeV}$ [1]. These reactions are considered an optimum reaction mechanism for neutron and radioactive ion beam (RIB) generation [2,3]. In both cases, an accurate understanding of this reaction mechanism is essential to reliably estimate the production rates of neutrons or RIBs, and also for the determination of the accumulated radiological inventory of the target assembly used in these facilities.

There have been important efforts during the last decade to collect high quality data for model benchmarking [4]. In particular, the isotopic production cross sections of final residual nuclei produced in spallation reactions were accurately determined at GSI, investigating these reactions in inverse kinematics by using a high-resolving power magnetic spectrometer. This technique made it possible to measure

\footnotetext{
*Corresponding author: carlos.PARADELADOBARRO@ec. europa.eu

${ }^{\dagger}$ Present address: Universidad de Vigo, E-36200 Vigo, Spain.

${ }^{\ddagger}$ Present address: GANIL, CEA/DSM-CNRS/IN2P3, BP 55027, F-14076 Caen, France.

${ }^{\S}$ Present address: Universidad de Santiago de Compostela, E-15782 Santiago de Compostela, Spain.

"Present address: Subatech (UMR 6457), CNRS/IN2P3-EMNUniversité de Nantes, 44307 Nantes, France.

"Present address: Chalmers University of Technology, SE-412 96 Gothenburg, Sweden.

"Present address: NSCL, East Lansing, MI 48824, USA.

${ }^{* *}$ Present address: Institute for Nuclear Research Nuclear Energy, Bulgarian Academy of Sciences, Sofia, Bulgaria.
}

reactions induced by ${ }^{238} \mathrm{U}$ [5-10], ${ }^{208} \mathrm{~Pb}$ [11-14], ${ }^{197} \mathrm{Au}$ [15-17], ${ }^{136} \mathrm{Xe}[18-20]$, and ${ }^{56} \mathrm{Fe}[21,22]$ projectiles on liquidhydrogen and liquid-deuterium targets at relativistic energies. These new data have contributed to improvement of the predictive power of model calculations describing spallation reactions.

The most important developments in model calculations include sophisticated models using transport equations [23], semiclassical models based in the impulse approximation [24], and even semiempirical codes [25]. Most of the efforts so far have been concentrated in the energy regime of the accelerators used for spallation neutron sources and RIB facilities, from 500 up to $1000 \mathrm{MeV}$. However, the use of thick spallation targets at these facilities implies a non-negligible contribution of secondary reactions at energies below $500 \mathrm{MeV}$ [1]. These secondary reactions contribute to neutron generation by means of $(p, p n)$ reactions [26], but also to the production of heavier residual nuclei by $(n, p)$ charge pickup reactions [27] or more neutron-rich ones by $(p, n)$ charge-exchange reactions [28].

Many of the existing data at low energies were obtained by using spectroscopic techniques, providing information on a limited number of final residual nuclei [29]. The complete identification of the final fragments using magnetic spectrometers is limited by the strong contribution of charge states at low energy, making difficult the unambiguous identification of the final nuclei. Recently, the isotopic composition of the spallation residual nuclei produced in reactions induced by ${ }^{137} \mathrm{Cs}$ and ${ }^{90} \mathrm{Sr}$ on hydrogen and deuterium at $185 \mathrm{~A} \mathrm{MeV}$ have been determined [30].

In this work, we propose to ameliorate the existing data set on residual nuclei production in spallation reactions at low energies by investigating the reaction ${ }^{136} \mathrm{Xe}+p$ at $200 A \mathrm{MeV}$. Moreover, these data will complete an energy systematics with our previous measurements at 500A [19] and $1000 \mathrm{~A} \mathrm{MeV} \mathrm{[18].}$ This set of data can be used to investigate the energy dissipation 
in spallation reactions, and, in particular, to validate the impulse approximation used in intranuclear cascade models at low energies.

\section{EXPERIMENTAL SETUP}

The experiment was performed at GSI (Darmstadt) using the fragment separator (FRS) spectrometer [31]. A detailed description of the setup can be found in Refs. [18,19]. The xenon beam was delivered by the synchrotron SIS and its intensity was continuously measured with the help of a secondary-electron monitor (SEETRAM) [32] consisting of three titatium foils of $13.5 \mathrm{mg} / \mathrm{cm}^{2}$. Then, as shown in Fig. 1, the primary beam impinged on a $87 \mathrm{mg} / \mathrm{cm}^{2}$ liquid-hydrogen target confined in a container with thin titanium windows of $36.3 \mathrm{mg} / \mathrm{cm}^{2}$ total thickness.

Because of the inverse kinematics, the residues produced in the reaction were emitted in the forward direction and they could be studied with the FRS. This is a two-stage magnetic spectrometer with dispersive intermediate (S2) and achromatic final (S4) focal planes. The FRS resolving power is $\Delta B \rho / B \rho \sim 1 / 1500$ with a momentum acceptance of $3 \%$ and an angular acceptance of $\pm 15 \mathrm{mrad}$ around the beam axis.

In Fig. 1 we also show the schematic drawing of all the detectors used in this work for the identification of the reaction residues. Two position-sensitive plastic scintillators [33] which are placed at both focal planes provide the magnetic rigidities and the time of flight (ToF) in the second FRS stage. It is worth mentioning that a thinner plastic scintillator has been used in this experiment compared to the one used with higher beam energies in order to reduce the energy loss of the fragments traversing the FRS. Multisampling ionization chambers (MUSICs) [34], placed in the final focal plane, are used to determine the atomic number of each fragment by measuring their energy loss in the filling gas. In our experiment the variations in the fragment atomic charge state are much more relevant than at higher beam energies. Therefore, three MUSIC detectors were placed in front of the Sc4 plastic scintillator to improve the nuclear charge resolution, when at higher energies one MUSIC is enough for the identification of nuclei with $Z$ around 50 [35].

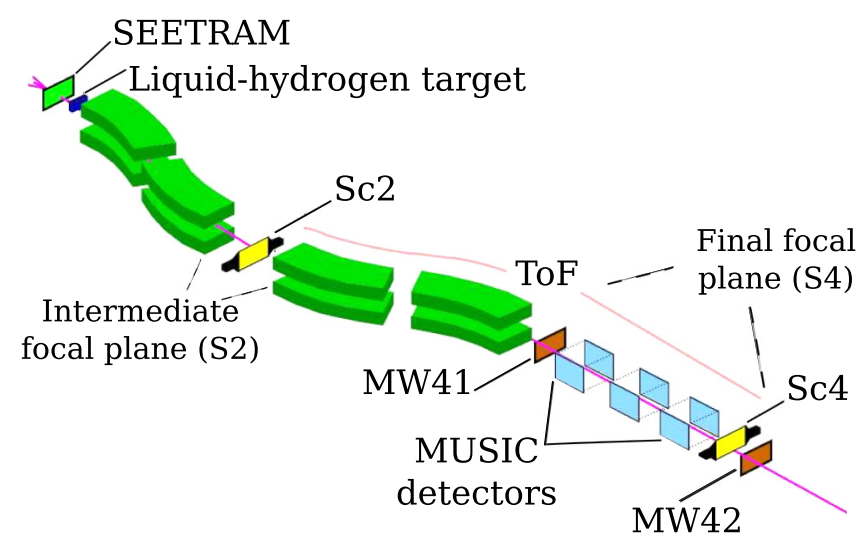

FIG. 1. Schematic drawing of the fragment separator with the detector equipment.
In addition to these detectors, several multiwire chambers (MW) [36], placed along the FRS, determine the fragment position for calibration purposes. They mainly consist of three planes of parallel wires: the central anode plane is made of $20 \mu \mathrm{m}$ tungsten wires separated by $2 \mathrm{~mm}$ and the cathode planes are made of $50 \mu \mathrm{m}$ wires separated by $1 \mathrm{~mm}$. The cathode planes are orthogonally placed to provide the twodimensional position, while the anode wires are rotated by $45^{\circ}$ with respect to the cathode wires. All the multiwire chambers are removed after the calibration runs except those placed at the final focal plane, MW41 and MW42. This information is relevant for the data analysis, as will be shown in Sec. III A 1.

In order to subtract the contribution of the reactions in the target windows, the SEETRAM monitor, and the accelerator exit window, the measurements were repeated with an empty container. Because of the limited momentum acceptance of the spectrometer and the selection in atomic number induced by the energy loss at S2, 13 different magnetic tunings for the FRS magnets, centering different iodine isotopes along the spectrometer, were required to cover all the residues having a significant production.

\section{DATA ANALYSIS}

\section{A. Residue identification}

The first goal of the analysis is to identify the fragments produced in the reaction that are transmitted through the FRS. The atomic number identification is based on the energy lost by the fragments in the three MUSIC detectors. The formation of atomic charge states degrades the resolution of the atomic number achieved with each individual MUSIC. However, as three MUSIC were included in the setup, all fragments pick up a similar number of electrons while traversing the total gas volume, reaching an equilibrium in the charge state distribution. Therefore, by considering the energy losses along the three MUSICs the separation between different fragments improves, as shown in Fig. 2.

On the other hand, the energy loss depends, according to the Bethe-Bloch expression, on the squared charge of the fragment and on its velocity. However, two effects modify this behavior in our case. First, the collection of electrons by the MUSIC anodes suffers from losses during the electron drift, producing a dependence with the dispersive coordinate $\left(x_{4}\right)$ of the fragments reaching the final image plane of the FRS. Furthermore, for fragment energies below $200 \mathrm{~A} \mathrm{MeV}$, the energy lost in the MUSICs becomes a significant fraction of the initial fragment energy. This means that the fragments reduce their velocity along the detector and this reduction also depends on the fragment mass. In the first step, previous to the mass identification, this correction was included in the fit, providing the fragment atomic number by means of a dependence on the mass corresponding to the iodine isotope that follows the central trajectory for each FRS setting, $A_{\text {cent }}$.

Taking into account these considerations, the atomic number $Z$ was derived using the following formula:

$\Delta E=P\left(x_{4}\right) Q(T o F) R(Z)\left[1+s_{0} A_{\text {cent }}\left(10^{-3} \times \Delta E\right)^{s_{1}}\right]$

where $\Delta \mathrm{E}$ accounts for the energy losses in each MUSIC, and $P, Q$, and $R$ are second-order polynomials on $x_{4}$, ToF, 


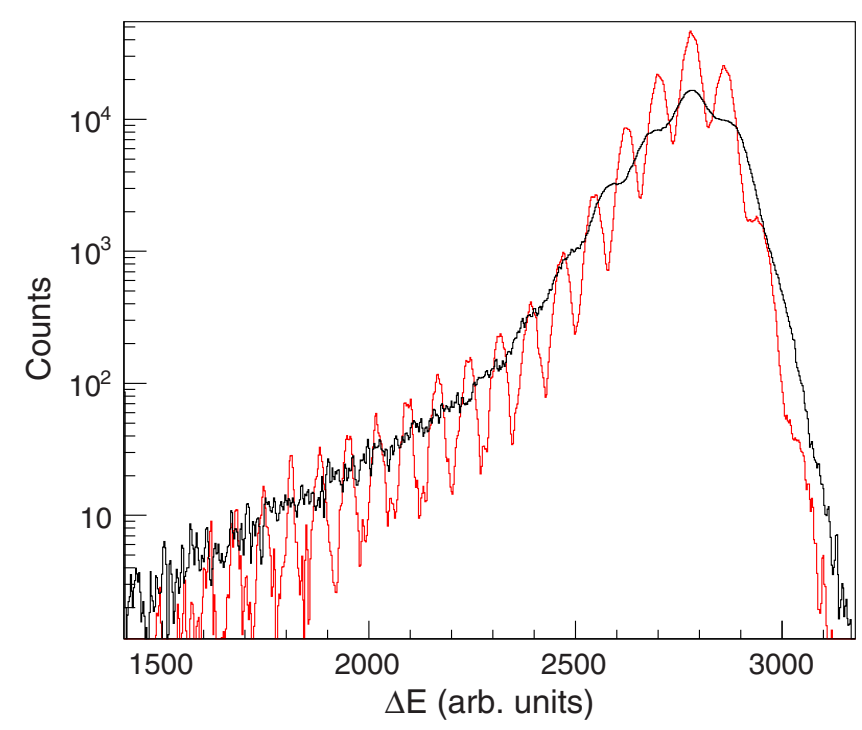

FIG. 2. Energy loss for the fragments transmitted in the FRS in the magnetic setting centered on ${ }^{131}$ I obtained with one MUSIC detector (black line) and combining the signals from the three MUSICs (red line).

and $Z$, respectively. The position of the fragment at the final image plane along the dispersive coordinate $\left(x_{4}\right)$ is provided by the scintillator placed after the ionization chambers ( $\mathrm{Sc} 4)$ and the ToF is obtained from the time difference between the two scintillators in the setup, which is directly related to the fragment velocity. Finally, the term with parameters $s_{0}$ and $s_{1}$ accounts for the dependence on the fragment mass. This formula allows us to determine a $Z$ value for each of the three MUSIC detectors. The polynomial parameters were determined by a fitting procedure where the phase space with coordinates $Z$, ToF, $x_{4}$, and $A_{\text {cent }}$ is divided into finite cells, and the average values of each coordinate in the cells are used. The results obtained for the three MUSIC detectors are calibrated using as reference the xenon beam and combined to get an accurate identification of the fragment atomic number. Once the mass is identified as explained below, it replaces $A_{\text {cent }}$ in Eq. (1), in order to provide the atomic number with an improved resolution of about $\Delta Z / Z=1 \times 10^{-2}$ full width at half maximum (FWHM), as shown in Fig. 3.

The mass identification of the residues is obtained from the determination of their magnetic rigidity according to the following expression:

$$
B \rho=\beta \gamma \frac{A}{q} c \frac{m_{0}}{e},
$$

where $B \rho$ is the magnetic rigidity of the fragments, $q$ their atomic charge, $\beta \gamma$ their relativistic velocity and the corresponding Lorentz factor, $c$ the speed of light, $m_{0}$ the nuclear mass unit, and $e$ the electron charge. Moreover, we need to assume that the residues are fully stripped $(q=Z)$ when traversing the FRS.

For measurements performed for the same reaction, but at higher energies $[18,19], A / Z$ was obtained by using the magnetic rigidity $\left(B \rho_{2}\right)$ of the fragments determined with the

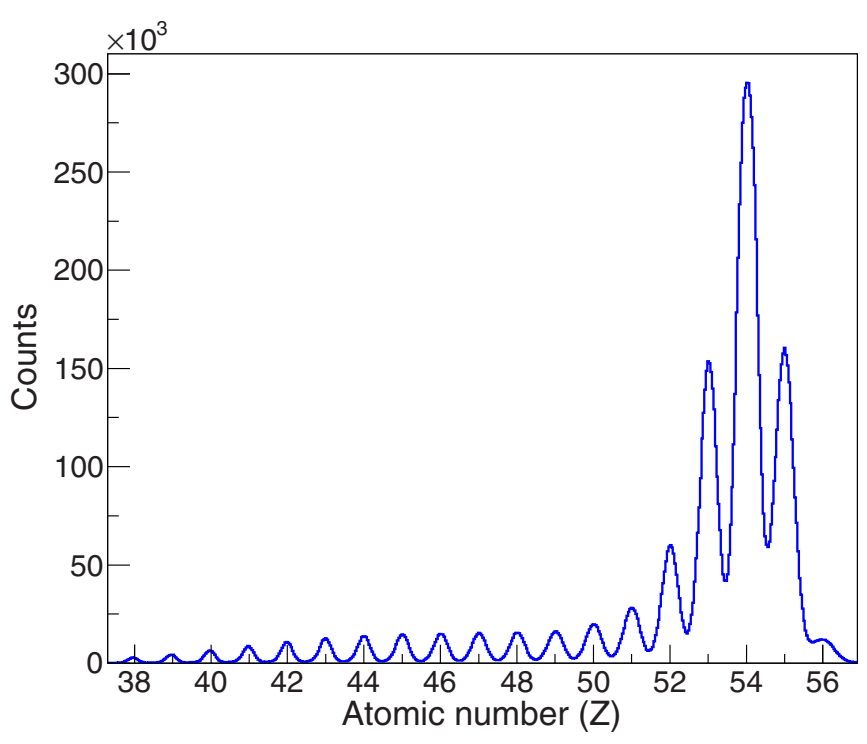

FIG. 3. Charge distribution of the reaction residues including all the measured FRS settings.

second part of the FRS according to the following expression:

$$
B \rho_{2}=B \rho_{02}\left(1+x_{2} / D_{2}+x_{4} / D_{4}\right)
$$

where $\rho_{02}$ is the radius of the central trajectory along the spectrometer and $D_{2}$ and $D_{4}$ are the optical dispersions of the first and the second stages, respectively. The positions along the dispersive coordinates at the intermediate and final image planes, $x_{2}$ and $x_{4}$, are measured by the plastic scintillators $\mathrm{Sc} 2$ and Sc4. These scintillators also provide the time of flight (ToF) between the two image planes and thus the fragment velocity. However, in our case, the mass resolution provided by the magnetic rigidity of the second part of the FRS was not as good as expected. Most probably this was due to the multiple scattering of the fragments in the detectors placed in front of the scintillator Sc4 one multiwire and three ionization chambers, deteriorating the measurement of the position at the final focal plane, $x_{4}$, and thus affecting the precise determination of $\rho_{2}$.

In order to overcome this limitation, an alternative method has been implemented. It consists of using the information of the first part of the FRS to improve the mass identification:

$$
B \rho_{1}=B \rho_{01}\left(1+x_{2} / D_{2}\right),
$$

where the accuracy for the magnetic rigidity is much better because of the more precise determination of the position of the trajectories at the intermediate image plane S2. The fragment velocity in the first part of the FRS can be reconstructed from the ToF measured between $\mathrm{S} 2$ and $\mathrm{S} 4$, correcting the velocity by the energy losses in the thin layers of matter at S2 for each $Z$.

As shown in Fig. 4, a large improvement in the mass resolution is achieved by applying the new method $\left[\Delta A_{1} / A_{1}=\right.$ $\left.2.6 \times 10^{-3}(\mathrm{FWHM})\right]$ instead of the standard one $\left[\Delta A_{2} / A_{2}=\right.$ $\left.6.5 \times 10^{-3}(\mathrm{FWHM})\right]$. Furthermore, the initial mass determination based on $x_{4}$ is still useful in order to remove the background due to reactions at the intermediate focal plane, as will be described in Sec. III A 3. 

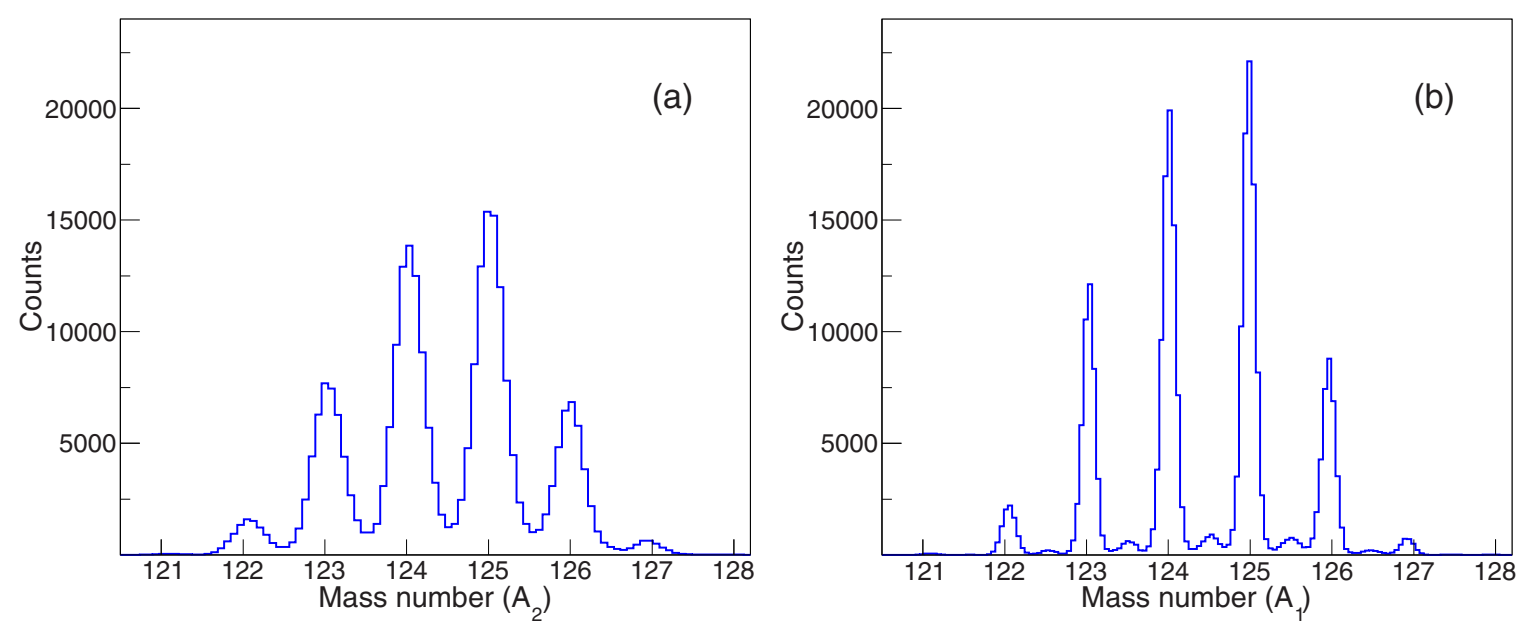

FIG. 4. Plot showing the improvement achieved in nuclear mass identification by using the second stage of the FRS (a) and the first stage (b). Data correspond to the iodine residues measured with the FRS magnetic setting centered on ${ }^{125}$ I.

The identification matrix obtained combining all the FRS magnetic settings is shown in Fig. 5, including more than 200 residual nuclei.

\section{Slowed-down fragment effect}

It can be observed in Fig. 4 that, when the mass resolution is improved by using Eq. (4), small peaks become visible in between the peaks associated with the integer mass numbers. Looking in detail the identification matrix displayed in Fig. 5, we also notice the presence of tails towards larger charge values for each residue spot, which correspond to the mentioned intermediate mass peaks. This effect can be clearly observed

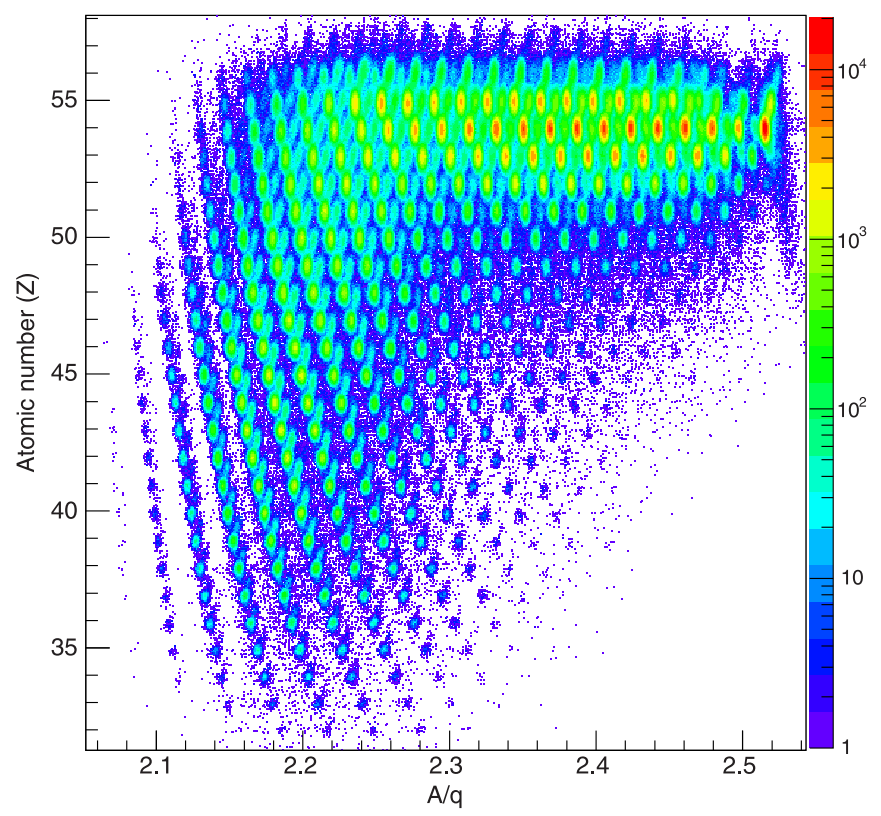

FIG. 5. Cluster plot showing the correlation between the atomic number $(Z)$ and the mass-over-charge ratio $(A / Z, Z)$ obtained from the 13 different FRS magnetic settings used in the experiment. It provides an unambiguous identification in atomic and mass number for the residues of the reaction ${ }^{136} \mathrm{Xe}+p$ at $200 A \mathrm{MeV}$. by studying the calibration runs without any target. For these runs, only the ${ }^{136} \mathrm{Xe}$ beam passes through the FRS, providing a reference value for the ion optics and the MUSICs' energy loss calibration. The corresponding identification plot is shown in Fig. 6. It can be observed that, even if most of the projectiles are properly identified, a significant amount is registered with an atomic number around 55, resulting in a tail already observed in the full identification matrix for each residue.

In order to explain this effect, we have suggested that the events in the tails correspond to those fragments traversing the cathode wires of the multiwire chamber MW41, which is permanently in the beam. This inhomogeneous layer of $50 \mu \mathrm{m}$ tungsten reduces the velocity of a significant part of the fragments after the FRS exit and before the first MUSIC. The slowed-down residues have significantly larger energy losses in the ionization chambers, while their measured ToF slightly

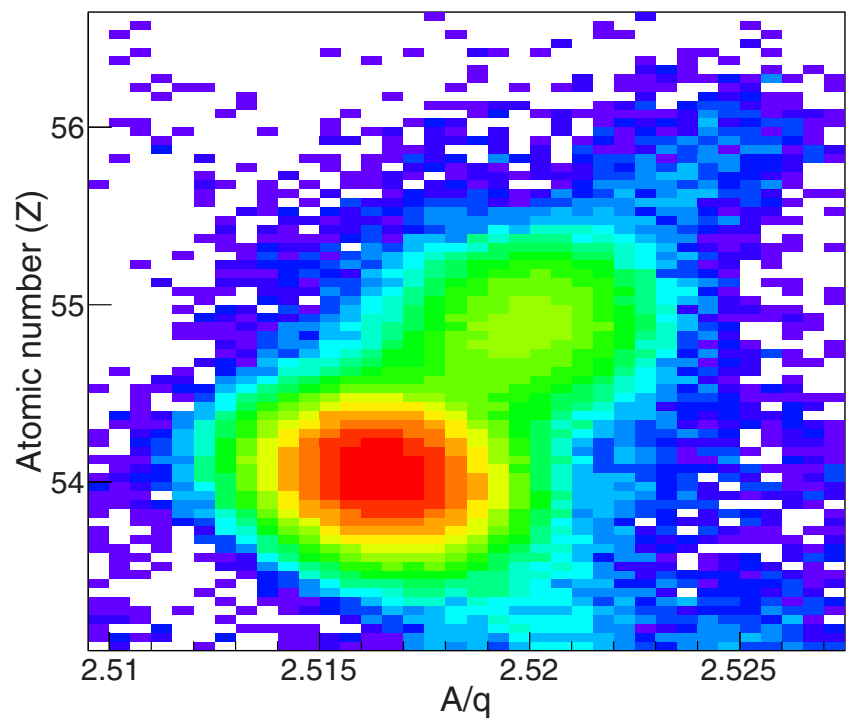

FIG. 6. Identification plot showing the particular shape of the beam spot, in which part of the beam particles are identified as $Z=55$ because of the lower velocity through the MUSICs. 
increase. The combined effect results in a misleading atomic and mass number identification. This hypothesis is supported by the fact that the number of events in the tail around $Z=55$ in Fig. 6 amounts up to the $9 \%$ of the total number of projectiles, which corresponds with the expected fraction of the beam slowed down by one of the cathode wires in MW41. Moreover, the number of events appearing as an additional tail around $Z=56$ in Fig. 6 is consistent with the number of primary beam projectiles slowed down by the intersection of the two cathode planes.

This is the first time that such an effect is described during a FRS experiment analysis, which can be explained due to the very low velocity of the primary beam. The estimated energy loss due to $50 \mu \mathrm{m}$ tungsten is about $5 \mathrm{MeV}$ per nucleon, which represents about $3 \%$ of the beam energy after traversing the FRS. This velocity reduction produces a variation in the stopping power, and thus in the energy loss in the MUSIC chambers, equivalent to the increase in one unit of the atomic number.

Finally, it can be observed in the identification matrix that these tails appear unambiguously separated from the closest $Z+1$ because of the different $A / Z$, and therefore the events in the tails can be assigned to the nuclei with the closest mass but one unit less in atomic number.

\section{Charge-state contamination}

A relevant contribution to background usually proceeds from the non fully stripped ions transmitted through the spectrometer: the so-called atomic charge states, $q$. $(A / Z)_{1}$ is in fact a measurement of $(A / q)_{1}$ in the first section of the FRS, whereas $(A / Z)_{2}$ would be $(A / q)_{2}$ in the second section. Therefore, the correlation between $(A / q)_{1}$ and $(A / q)_{2}$ is expected to show three diagonals: the main one containing those events keeping the same charge state through the full spectrometer $\left(q_{1}=q_{2}=Z\right.$ and $\left.q_{1}=q_{2}=Z-1\right)$, another one containing events picking one electron at $\mathrm{S} 2\left(q_{1}=Z, q_{2}=\right.$ $Z-1)$, and, on the other side, a third diagonal containing events stripped at $\mathrm{S} 2\left(q_{1}=Z-1, q_{2}=Z\right)$ [37]. However, in Fig. 7 we can only observe the main diagonal and some background events close to it, whereas the fragments changing its charge state at $\mathrm{S} 2$ are absent. The reason is that the $B \rho$ variation induced by one unit charge in the atomic charge state is of the order of $1 / 50=2 \%$ larger than FRS $B \rho$ acceptance $( \pm 1.5 \%)$. In any case, those events out of the main diagonal have been removed with software cuts in order to reject any background contribution due to atomic charge states from the secondary diagonals. In Sec. III B, we will determine the probabilities of the different atomic charge state configurations which are important for determining the cross sections.

Finally, the discrimination of the nonstripped ions keeping one electron all along the spectrometer $\left(q_{1}=q_{2}=Z-1\right)$, which contaminate the main diagonal, still remains. If one notes $P_{1}$ as the probability of an ion with $1 e^{-}\left(q_{1}=Z-1\right)$ in the first FRS section and $P_{2}$ as the same probability but for the second section of the FRS, the probability for an ion keeping one electron along the FRS, $P_{1} P_{2}$, is usually small compared to the probability of traversing the FRS fully stripped, $\left(1-P_{1}\right)\left(1-P_{2}\right)$. In order to calculate these

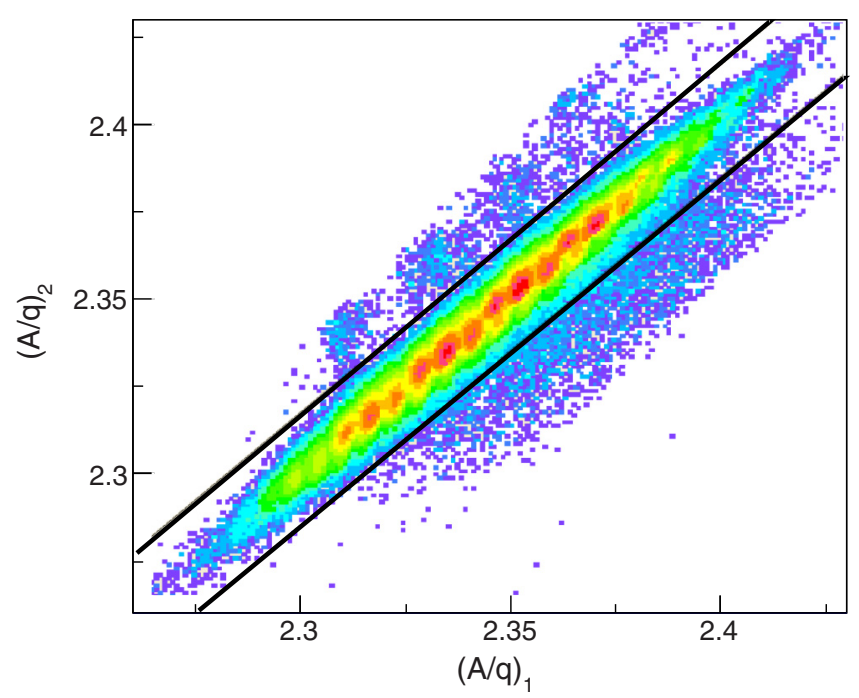

FIG. 7. Plot showing the correlation between the mass identification obtained in the two FRS sections. It allows the rejection of the background due to secondary reactions before the MUSICs (beyond the solid lines).

probabilities, we have used the GLOBAL code [38]. As can be observed in Fig. 8 , the $1 e^{-}$probabilities decrease with the fragment atomic number, so that in the first section of the FRS the highest values are $P_{1}=0.20$ and $P_{2}=0.15$ for $Z=54$. Therefore, the largest contamination due to nonstripped fragments amounts up to $3 \%$. However, even remaining at the few percent level, such type of contamination can be significant for the most neutron-rich residues because they are contaminated by the charge states of nuclei with $A-3$, having

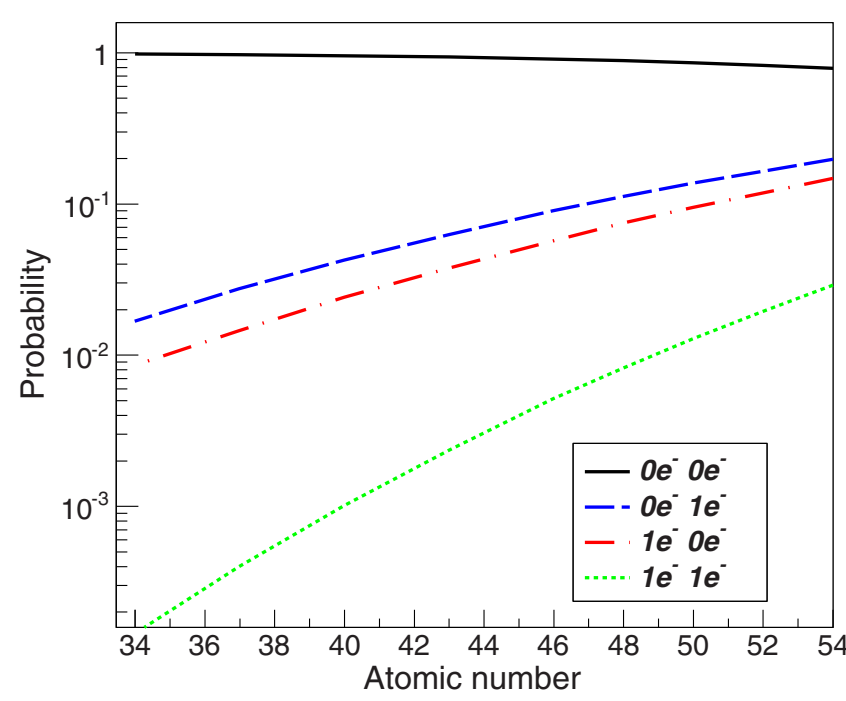

FIG. 8. Charge state probabilities predicted by the GLOBAL code for fragments produced in the reaction $\mathrm{Xe}+p$ at $200 \mathrm{~A} \mathrm{MeV}$ when traversing each FRS stage. The solid (black) curve refers to the probability of having fully stripped fragments in both stages $\left(q_{1}=\right.$ $q_{2}=Z$ ); dashed (blue) curve: $q_{1}=Z, q_{2}=Z-1$; dashed-dotted (red) curve: $q_{1}=Z-1, q_{2}=Z$; and dotted (green) curve: $q_{1}=$ $q_{2}=Z-1$. 


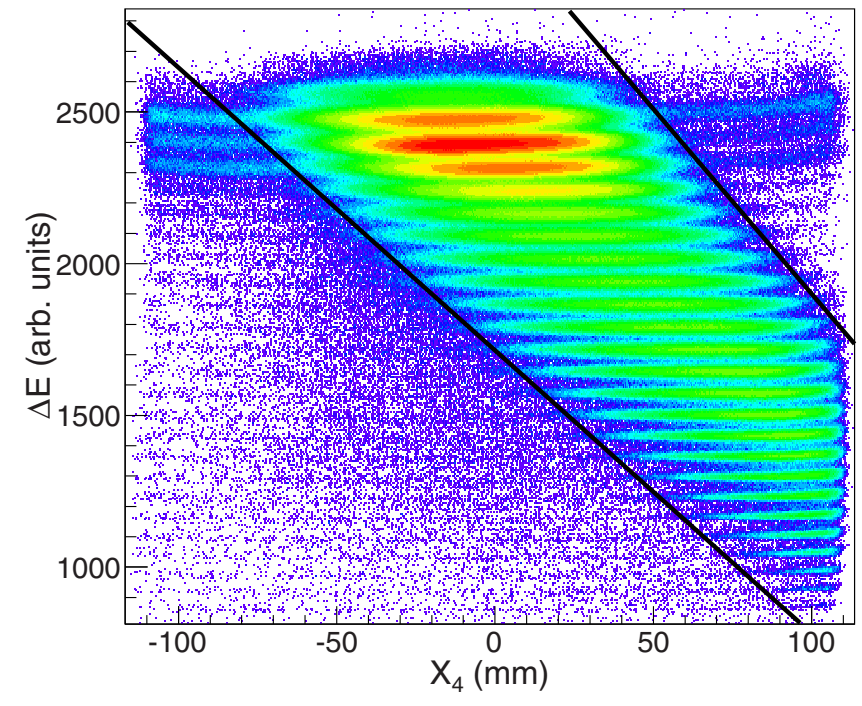

FIG. 9. The figure shows the background due to secondary reactions in S4 before the MUSICs for the FRS setting centered on ${ }^{131}$ I. Solid lines show the selection applied to reject the fragments that interact just before reaching the MUSICs.

a much higher production cross section [28]. In any case, the contributions due to the charge states are smaller than $20 \%$ and they have been corrected according to the GLOBAL prediction.

\section{Background reduction}

Figure 9 shows the correlation between the measured position at the final focal plane of the spectrometer and the reconstructed fragment atomic number for a setting of the FRS centered on ${ }^{131} \mathrm{I}$. It can be observed that the events are essentially located in a diagonal zone, as expected from the energy loss in the scintillator at S2 which mainly depends on $Z$. In fact, because of the spectrometer achromatism, all events would lie around $x_{4}$ center if no energy loss occurs at S2. However, we can also observe that an important scattered background is present on the left of the diagonal. This background can be explained as secondary reactions taking place before the first MUSIC detector, mainly originating from fragments with high atomic number reacting in the FRS vacuum window and the multiwire MW41. The discrimination of these events is important for the analysis because the expected production of low charge fragments at $200 \mathrm{~A} \mathrm{MeV}$ is really suppressed when compared to the production of residues close to the projectile. A good background rejection is achieved by requiring the conditions detailed in Figs. 7 and 9 .

\section{B. Production cross sections}

Once each residue is unambiguously identified in atomic and mass numbers by contours in the identification matrix, we can determine with high accuracy their velocity at the FRS entrance by using the magnetic rigidity in the first stage according to the following expression:

$$
\beta \gamma \propto \frac{(B \rho)_{1}}{A / Z},
$$

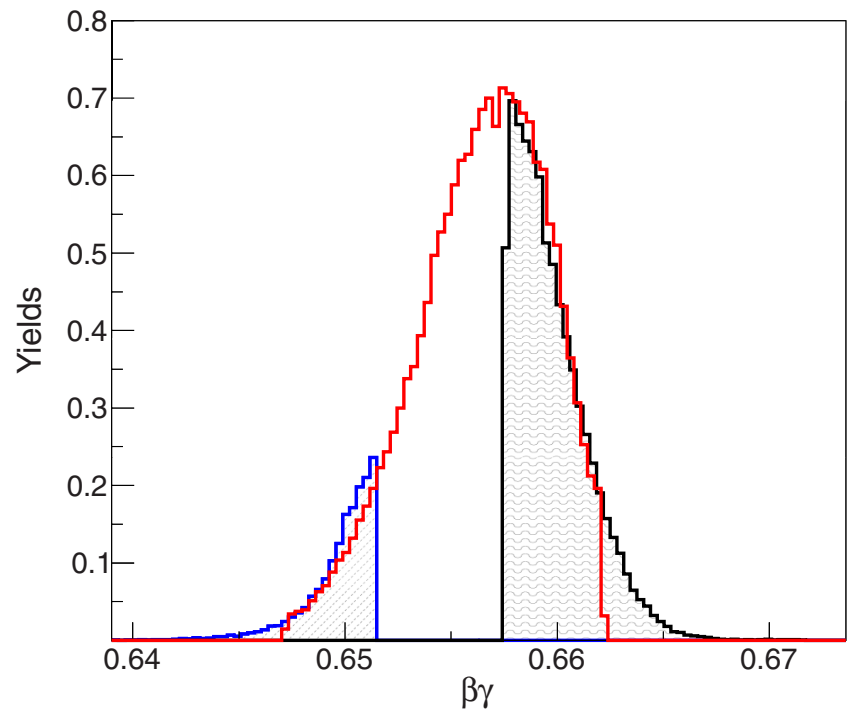

FIG. 10. Reconstruction of momentum distribution of the ${ }^{125} \mathrm{Xe}$ fragments using three different FRS magnet settings.

where the masses and the atomic numbers are considered as integers. If we combine the different magnetic settings of the FRS for which a specific fragment is transmitted, its momentum distribution can be reconstructed, as shown in Fig. 10.

In this type of experiment, the measured quantity is the differential yield relative to the longitudinal momentum of the fragments produced in the reaction. The total production yield for each identified residue is obtained by integrating the momentum distribution. We can then determine the production cross section by normalizing to the number of incident projectiles and the number of atoms per surface unit in the target. The contribution due to reactions with the target windows is determined by the measurement with the empty target. Figure 11 shows the element production with the filled (open circles) and the empty (solid circles) target. The contribution of the target container is almost negligible for fragments with atomic and mass numbers close to xenon. However, it becomes the dominant contribution for light residues far away from the projectile.

In addition, the following corrections were applied to take into account the losses due to the experimental method:

(i) The dead time of the data acquisition, which was kept between 10 and $35 \%$ and with an assigned uncertainty of $1 \%$.

(ii) The angular acceptance of the FRS, which is negligible for the fragments close to the beam but reaches about $25 \%$ for the lightest ones [18,20,39].

(iii) The fraction of non fully stripped fragments traversing the FRS, which was evaluated by using the GLOBAL code [38]. At this beam energy, only $65 \%$ of the nuclei with the largest values of atomic number traverse, fully stripped, both FRS stages, as shown in Fig. 8.

(iv) The fraction of fragments lost by nuclear reactions at the intermediate focal plane and with the matter layers 


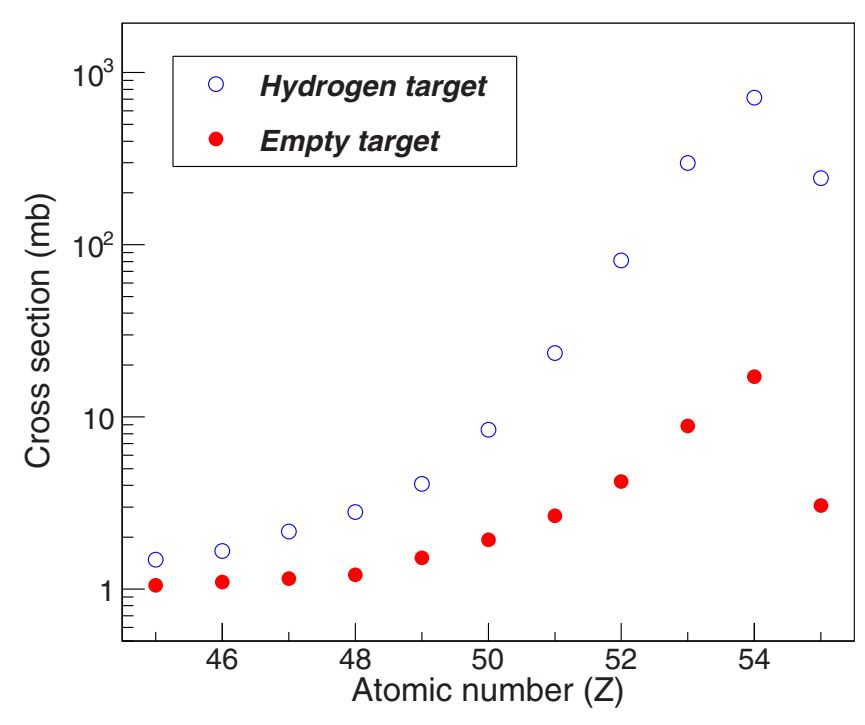

FIG. 11. Isobaric production yields obtained with the liquidhydrogen target in the titanium container (open circles) and with the empty titanium container (solid circles).

at the final focal plane before the first MUSIC, which amounts up to $1.5 \%$ for the heaviest nuclei.

(v) The multiple reactions within the target, which are corrected by using an algorithm describing the different probabilities for each nucleus to suffer a second reaction in the target and to be produced through an intermediate nucleus. This algorithm makes use of the empirical parametrization of projectile-fragmentation cross sections (EPAX) [40] and the microscopic model developed by Karol [41]. These multiple reactions in the target depopulate the production yields of heavy residual nuclei while they increase the production yields of the lighter fragments.

Because the statistical uncertainty of the production yields is kept below $2 \%$ for most of the residues measured in this work, the final uncertainty of the production cross sections is governed by systematic uncertainties. The systematic uncertainty associated with the number of incident projectiles provided by the SEETRAM calibration was estimated to be about $7 \%$. The thickness of the hydrogen target was carefully investigated in previous experiments with an estimated uncertainty of 3\% [5]. The other contributions to the systematic uncertainties of the measured yields were due to the accuracy of the different correction factors we have applied. The largest one is due to the estimation of the production of the ionic charge states using the GLOBAL code, which amounts to up to $9 \%$ for the heaviest nuclei and decreases down to $4 \%$ for the lightest ones. Finally, the uncertainty due to the multiple reactions is below $1 \%$, and the transmission correction uncertainty is only significant in a few cases where the velocity distribution is incomplete. All other uncertainties remain below 2\%. Taking into account that all these contributions add quadratically, the total uncertainties range from $10 \%$ to $15 \%$.

\section{RESULTS AND BENCHMARKING OF MODEL CALCULATIONS}

In this work we were able to determine the production cross sections of 91 residual nuclei produced in the studied reaction. These cross sections, together with their corresponding uncertainties, are listed in Table I. The yields obtained for the isotopes with masses below 115 endure from an incomplete correction for multiple reactions in the target and they could not be precisely determined in this work. Their contribution, being in total smaller than $0.5 \mathrm{mb}$ according to model calculations, can be neglected when accounting for the integral cross section.

The measured production cross sections cover almost completely the isotopic distributions from cesium to tin, as shown in Fig. 12. However, the iodine residues with mass number 134 and 135 could not be measured because they were not significantly transmitted in any FRS magnetic setting. In order to provide a realistic comparison with the predicted total reaction cross section, their contribution has been estimated from the results obtained for the same reaction at $500 \mathrm{~A} \mathrm{MeV} \mathrm{[19]} \mathrm{and} 1000 \mathrm{~A} \mathrm{MeV} \mathrm{[18],} \mathrm{taking} \mathrm{advantage}$ of the fact that the proton removal channel production is practically independent of the reaction energy, as shown in Ref. [42]. Moreover, extrapolated values for the production of ${ }^{131} \mathrm{Te},{ }^{132} \mathrm{Te}$, and ${ }^{128} \mathrm{Sb}$ isotopes have also been used in order to allow a reliable comparison with the codes. The estimated production cross sections for these iodine, tellurium, and antimony residues are represented by open circles in Fig. 12 and, including them, the integral cross section obtained is 1405 $( \pm 166) \mathrm{mb}$. This result is larger than the $1285 \mathrm{mb}$ obtained from the Karol model [41] but still compatible within the experiment uncertainty.

Figure 13 represents the measured cross sections, including the extrapolated ones, as a function of the fragment mass number. The results show that the fragment production is strongly suppressed for masses below $A=127$, with a maximum in the production around the mass number 130.

\section{A. Model calculations}

To complete the discussions presented in previous works concerning the investigation of the same reaction but at higher energies $[18,20]$, the cross sections measured in this experiment have been compared with simulation codes commonly used to describe spallation reactions. Spallation reactions are explained as a two-step process [43]. The first stage is often described as an intranuclear cascade: a sequence of quasifree nucleon-nucleon collisions where the resulting nucleus, the so-called prefragment, gains certain excitation energy. In the second stage, the deexcitation of the prefragment is described following statistical models. In the literature, there are different codes to describe each stage of the reaction. In this work, we have employed two different intranuclear cascade codes, ISABEL [44,45] and INCL4.6 [46], combined with the statistical deexcitation code ABLA07 [47].

The ISABEL model describes the interaction between protons and nuclei within relativistic classical mechanics. The nucleons of the nucleus are treated as a continuous medium 
TABLE I. Isotopic cross section of the residues produced in collisions induced by ${ }^{136} \mathrm{Xe}$ at $200 \mathrm{~A} \mathrm{MeV}$ with protons. Uncertainties are related providing the last two significant figures between parentheses. Asterisks indicate estimated cross sections (see text for details).

\begin{tabular}{|c|c|c|c|c|c|}
\hline$Z$ & $A$ & $\sigma(\mathrm{mb})$ & $Z$ & $A$ & $\sigma(\mathrm{mb})$ \\
\hline 55 & 136 & $4.19(50)$ & 54 & 135 & 87.7 (100) \\
\hline 55 & 135 & 13.7 (16) & 54 & 134 & $73.6(87)$ \\
\hline 55 & 134 & $21.8(26)$ & 54 & 133 & $68.4(81)$ \\
\hline 55 & 133 & $29.0(34)$ & 54 & 132 & $71.2(84)$ \\
\hline 55 & 132 & $28.7(34)$ & 54 & 131 & $69.4(82)$ \\
\hline 55 & 131 & $28.3(34)$ & 54 & 130 & $70.1(83)$ \\
\hline 55 & 130 & $25.6(30)$ & 54 & 129 & $63.9(76)$ \\
\hline 55 & 129 & $24.0(28)$ & 54 & 128 & $60.0(72)$ \\
\hline 55 & 128 & $22.0(26)$ & 54 & 127 & $50.8(60)$ \\
\hline 55 & 127 & $19.0(22)$ & 54 & 126 & $42.5(50)$ \\
\hline 55 & 126 & $13.9(16)$ & 54 & 125 & $31.5(37)$ \\
\hline 55 & 125 & $9.6(11)$ & 54 & 124 & $19.6(23)$ \\
\hline 55 & 124 & $5.16(61)$ & 54 & 123 & $10.2(12)$ \\
\hline 55 & 123 & $1.94(23)$ & 54 & 122 & $3.42(41)$ \\
\hline 55 & 122 & $0.387(48)$ & 54 & 121 & $0.566(76)$ \\
\hline 55 & 121 & $0.0320(46)$ & 54 & 120 & $0.00285(52)$ \\
\hline 53 & 135 & $20.4(*)$ & 52 & 132 & $1.0(*)$ \\
\hline 53 & 134 & $19.1(*)$ & 52 & 131 & $2.0(*)$ \\
\hline 53 & 133 & $20.2(22)$ & 52 & 130 & $3.15(33)$ \\
\hline 53 & 132 & $21.8(24)$ & 52 & 129 & $3.62(38)$ \\
\hline 53 & 131 & $27.3(30)$ & 52 & 128 & $5.47(57)$ \\
\hline 53 & 130 & $27.3(30)$ & 52 & 127 & $6.68(70)$ \\
\hline 53 & 129 & $29.0(32)$ & 52 & 126 & $7.06(74)$ \\
\hline 53 & 128 & $31.3(35)$ & 52 & 125 & $8.38(87)$ \\
\hline 53 & 127 & $32.5(36)$ & 52 & 124 & $9.13(95)$ \\
\hline 53 & 126 & 29.7 (33) & 52 & 123 & $9.02(94)$ \\
\hline 53 & 125 & $24.5(27)$ & 52 & 122 & $8.45(89)$ \\
\hline 53 & 124 & $20.0(22)$ & 52 & 121 & $6.58(69)$ \\
\hline 53 & 123 & $13.9(15)$ & 52 & 120 & $4.36(46)$ \\
\hline 53 & 122 & 8.90 (99) & 52 & 119 & $2.00(22)$ \\
\hline 53 & 121 & $4.83(55)$ & 52 & 118 & $0.655(78)$ \\
\hline 53 & 120 & $2.01(30)$ & 52 & 117 & $0.162(22)$ \\
\hline \multirow[t]{2}{*}{53} & 119 & $0.478(78)$ & & & \\
\hline & & & 50 & 123 & $0.200(20)$ \\
\hline 51 & 128 & $0.3(*)$ & 50 & 122 & $0.314(31)$ \\
\hline 51 & 127 & 0.645 (64) & 50 & 121 & $0.433(42)$ \\
\hline 51 & 126 & $0.788(78)$ & 50 & 120 & $0.580(57)$ \\
\hline 51 & 125 & $1.38(14)$ & 50 & 119 & $0.679(67)$ \\
\hline 51 & 124 & $1.67(16)$ & 50 & 118 & $0.730(72)$ \\
\hline 51 & 123 & $2.22(22)$ & 50 & 117 & $0.641(64)$ \\
\hline 51 & 122 & $2.34(23)$ & 50 & 116 & $0.490(49)$ \\
\hline 51 & 121 & $2.68(26)$ & 50 & 115 & $0.344(36)$ \\
\hline 51 & 120 & $2.23(22)$ & & & \\
\hline 51 & 119 & $1.91(19)$ & 49 & 120 & $0.0806(91)$ \\
\hline 51 & 118 & $1.36(14)$ & 49 & 119 & $0.109(12)$ \\
\hline 51 & 117 & $0.882(91)$ & 49 & 118 & $0.146(15)$ \\
\hline 51 & 116 & $0.463(50)$ & 49 & 117 & $0.204(22)$ \\
\hline \multirow[t]{2}{*}{51} & 115 & $0.277(32)$ & 49 & 116 & $0.209(21)$ \\
\hline & & & 49 & 115 & $0.216(21)$ \\
\hline 48 & 116 & $0.0511(69)$ & & & \\
\hline 48 & 115 & 0.0687 (97) & & & \\
\hline
\end{tabular}

or Fermi sea inside a potential well. The nuclear density distributions are described by a steplike function divided into sixteen regions of constant density fitted to a Yukawa potential including diffuse boundaries. The cascade particles are followed until they leave the volume, or their energy falls below a certain cutoff energy, which corresponds to the Coulomb barrier plus twice the binding energy. Pauli blocking is also taken into account, excluding the cascade particles with an energy below the Fermi level. The excitation energy of the prefragments is determined according to the sum of the holes and particle energies which fall below the cutoff energy.

In the INCL4.6 model, the proton-nucleus collision is modeled as a sequence of binary collisions between the nucleons of the system. The nucleus is represented by a potential well according to a Woods-Saxon distribution, whose radius depends on the nucleon momentum [24]. The isospin and energy dependences of the potentials are calculated according to optical models [46], and the Pauli blocking is considered by means of statistical factors [24]. Nucleons move along straight trajectories until they undergo a collision with another nucleon or until they reach the surface, where they eventually escape. The excitation energy of the prefragments is obtained from the difference between the particle kinetic energies of the initial and final states. The latest version of INCL also includes a dynamical coalescence algorithm for the production of light clusters (up to masses of $A=8$ ).

In a second step, the prefragment deexcites by means of a sequential evaporation of particles. The statistical code ABLA07 describes the deexcitation of a nonfissile nucleus emitting $\gamma$ rays, neutrons, light charged particles, and intermediate-mass fragments (IMFs) according to Weisskopf's formalism [48]. Particle separation energies and emission barriers for charged particles are considered according to the experimental mass evaluation from 2012 [49] and the Bass potential [50], respectively. The deexcitation by fission is also included according to a dynamical picture described in Refs. [51,52].

These model calculations have been benchmarked at high energies, providing a satisfactory description of many observables [8,20,22,53-58]. However, at low energies, close to the spallation regime limit of $150 \mathrm{MeV}$, these calculations have never been confronted with complete isotopic-fragment distributions.

In Fig. 12 the isotopic distributions of the production cross sections corresponding to elements from cesium to cadmium are compared to the predictions obtained with these codes. In general, both codes provide an acceptable description of the shape of the isotopic distribution cross sections, but they fail when estimating their absolute values. In particular, they significantly underestimate the production of nuclei with mass numbers around 130 for the xenon fragments, which represent a major contribution to the integral cross section. It is worth mentioning that ISABEL (dashed lines) reproduces better the isotopic distribution of cesium residues, the charge-exchange channel. However, for fragments with atomic number smaller than the projectile, INCL4.6 model (solid lines) seems to describe the cross sections much better than ISABEL. This fact could be expected because ISABEL does not include the emission of light clusters [44] during the cascade process. The 

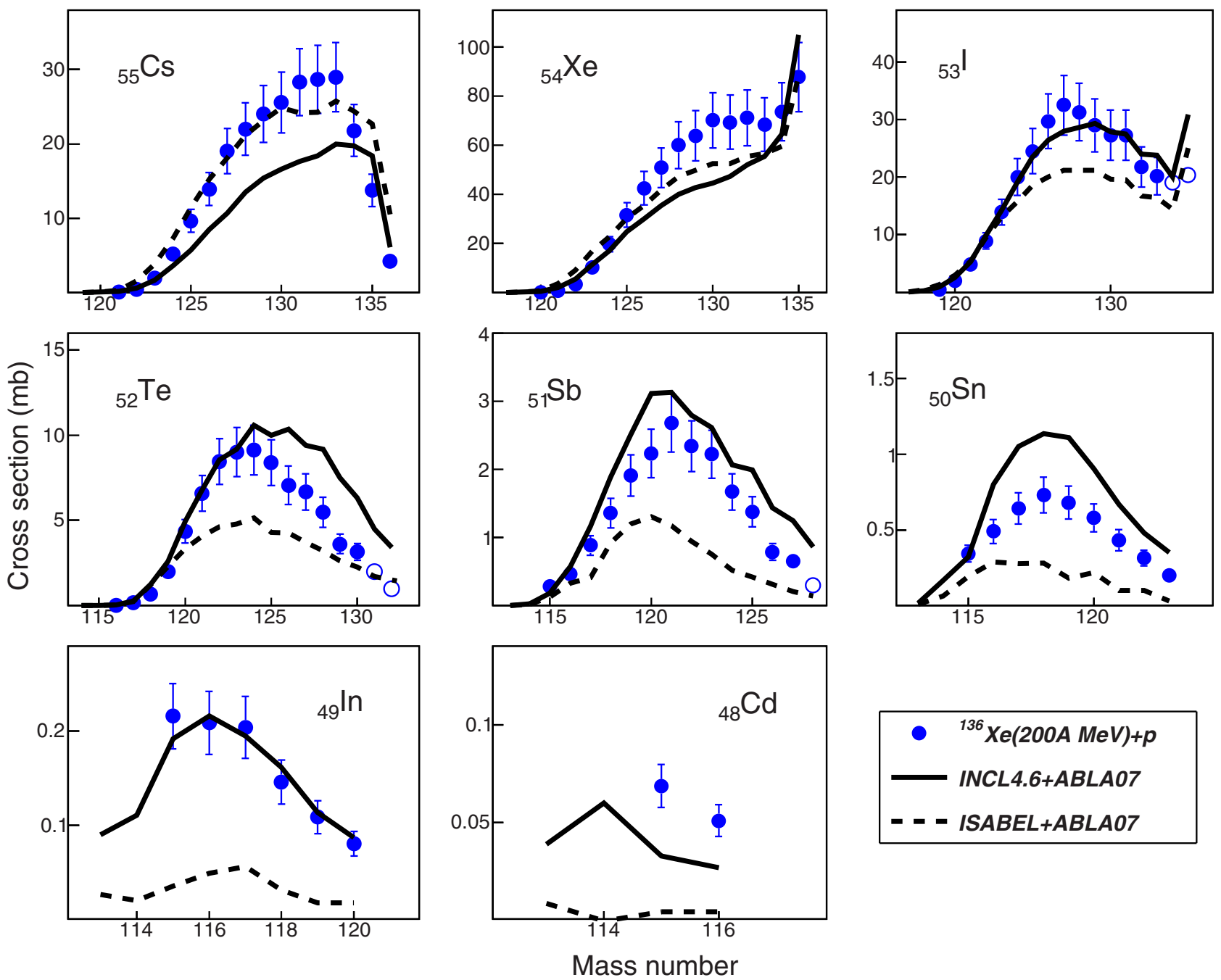

FIG. 12. Isotopic distributions of the production cross sections measured in this work compared to the predictions of the codes INCL4.6 (solid lines) and ISABEL (dashed lines) coupled to the deexcitation code ABLA07. Solid circles represent measured data while empty circles are the extrapolated ones.

emission of these clusters produces lighter residual nuclei, increasing their cross sections.

As for the isotopic cross section, in Fig. 13 an underestimation of the isobaric cross section in the region of residual nuclei around $A=130$ is clearly observed while for $A=135$ both calculations overestimate the measurements. Below $A=125$ both codes provide a reasonable description of the isobaric cross sections, however for ISABEL this result is a compensation effect because the isotopic distributions are not properly described.

The observed underestimation of the production of heavyresidual nuclei indicates an incomplete description of the most peripheral collisions where these nuclei are produced. A possible explanation would be related to the restriction in the INCL codes to only two-body, $N-N$, collisions without considering mean field contributions. In this approach, collective effects due to mean-field resilience are disregarded, resulting in a reduced stability of the heavy remnants. In addition, an incomplete description of the proton and neutron abundances at the nucleus surface may play a relevant role. In this context, some efforts have recently been made using INCL [42,59], with the most relevant aspects being the neutron skin effects and the correlation between the energy of the nucleon orbitals and their radius.

\section{B. Energy dependence of the production yields of spallation residues}

The main aim of the experimental campaign on the ${ }^{136} \mathrm{Xe}+p$ reaction at the FRS facility was to investigate the energy dissipation during the first stage of the collision by comparing measurements at different projectile energies. This energy dependence of the residue production becomes important for thick spallation targets, where the high energy primary protons can be significantly slowed down before inducing a spallation reaction with the heavy nuclei in the target, or secondary reactions may occur. Therefore, it is crucial for the benchmark of thick target simulations to obtain results on the same system at different energies, in particular, 


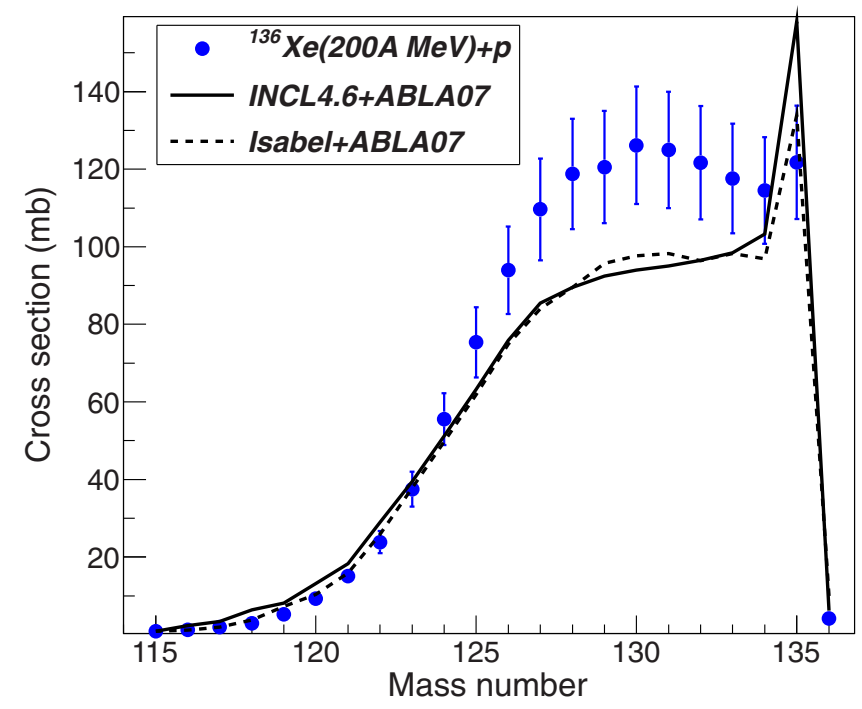

FIG. 13. Isobaric cross section distribution obtained for the reaction ${ }^{136} \mathrm{Xe}+p$ at $200 \mathrm{~A} \mathrm{MeV}$, compared to the results provided by the simulation codes INCL4.6 (solid line) and ISABEL (dashed line), both coupled to the deexcitation code ABLA07.

at energies as low as $200 \mathrm{~A} \mathrm{MeV}$ where the only open channel for nucleon-nucleon collisions is the elastic one.

The first evidence obtained from this work is that the total cross section measured at $200 \mathrm{~A} \mathrm{MeV}$ is compatible with those measured at $500 \mathrm{~A}$ and $1000 \mathrm{~A} \mathrm{MeV}$, as shown in Table II. As predicted from Karol's formula [41], the experimental results do not show a clear trend of the total reaction cross section with the energy. On the contrary, predictions obtained with intranuclear cascade codes show a decreasing trend of the total cross sections with energy.

On the other hand, the shape of the isobaric-fragment distributions varies drastically with the incident energy, as can be observed in Fig. 14. We can then conclude that total reaction cross sections are mostly governed by the sizes of the projectile and target nuclei while the residual fragment distribution seems to be sensitive to the energy dissipated in the collision.

As observed in Fig. 14, the progressive reduction in the production of the lightest residues with decreasing incident energies is compensated by the increment in the production of heavier residues. This evolution can be explained by the dependence of the dissipated energy on the initial kinetic energy of the projectiles. The fraction of kinetic energy dissipated into internal excitation of the reaction remnant

TABLE II. Measured total reaction cross section induced by ${ }^{136} \mathrm{Xe}$ projectiles on proton at $200 \mathrm{~A}, 500 \mathrm{~A}$, and $1000 \mathrm{~A} \mathrm{MeV}$ compared to the predictions obtained with INCL4.6 and ISABEL coupled to ABLA07 and with the Karol model.

\begin{tabular}{lcccc}
\hline \hline Energy $(A \mathrm{MeV})$ & Experiment & INCL & ISABEL & Karol \\
\hline 1000 & $1393(72)$ & 1390 & 1335 & 1353 \\
500 & $1388(97)$ & 1265 & 1253 & 1263 \\
200 & $1405(166)$ & 1242 & 1193 & 1285 \\
\hline \hline
\end{tabular}

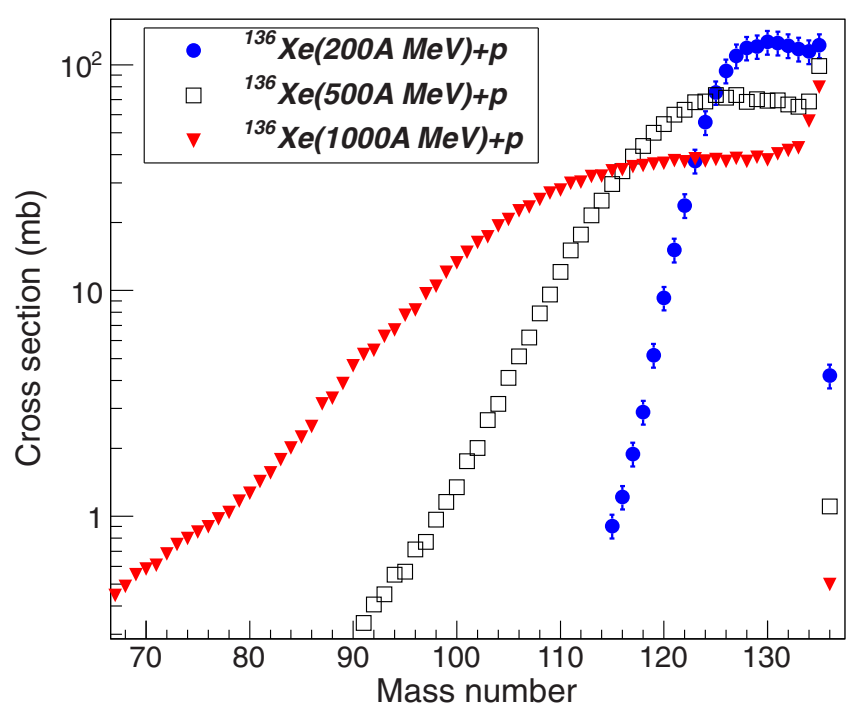

FIG. 14. Isobaric distributions of the measured cross sections for the ${ }^{136} \mathrm{Xe}+p$ reactions at $1000 A \mathrm{MeV}$ (solid triangles), $500 A \mathrm{MeV}$ (open squares), and $200 \mathrm{~A} \mathrm{MeV}$ (solid circles)

clearly increases with the projectile energy. The evacuation of this excitation energy by nucleon or cluster evaporation will then lead to longer evaporation chains at high excitation energies, producing lighter residual nuclei. The dependence of the final fragment on the excitation energy gained by the cascade prefragments is extensively discussed in Ref. [20].

Figure 15 shows the evolution of the isotopic distributions of the residual fragments with the projectile energy. For the production of cesium isotopes in charge exchange reactions, it can be observed that the shape of the isotopic distribution for the three energies is very similar, with the production being more important at $200 \mathrm{~A} \mathrm{MeV}$, approximately four times larger than at $500 \mathrm{~A} \mathrm{MeV}$, and ten times more than at $1000 \mathrm{~A} \mathrm{MeV}$. This behavior could be expected because the charge-exchange reaction at $200 \mathrm{~A} \mathrm{MeV}$ is exclusively elastic while at higher energies the excitation of nucleon resonances implies the dissipation of a large amount of energy decreasing the survival probability of the remnants. The increase on the inelastic nucleon-nucleon cross section explains the observed reduction of the cross section of cesium isotopes with energy.

For the xenon residues we observe a similar energy dependence as for the cesium. Again the role of the inelastic nucleon-nucleon processes could explain this result. The shape of the isotopic distribution is rather similar at $500 \mathrm{~A}$ and $1000 A \mathrm{MeV}$ but at $200 \mathrm{~A} \mathrm{MeV}$ is clearly narrower. Indeed, the production of the most neutron-deficient isotopes is strongly suppressed at $200 \mathrm{~A} \mathrm{MeV}$.

For the elements lighter than xenon we observe a fast reduction of the cross sections corresponding to residual nuclei produced at $200 \mathrm{~A} \mathrm{MeV}$. We also observe a clear shift of the highest production towards more neutron-rich residues as compared to the distributions measured at higher incident energies.

Finally, we compare these three energies in terms of the average neutron excess of the isotopic distributions $\langle N\rangle / Z$ in Fig. 16. From the results at high energies, the existence of a rather universal pattern dominated by the deexcitation process of the prefragments produced in the intranuclear 

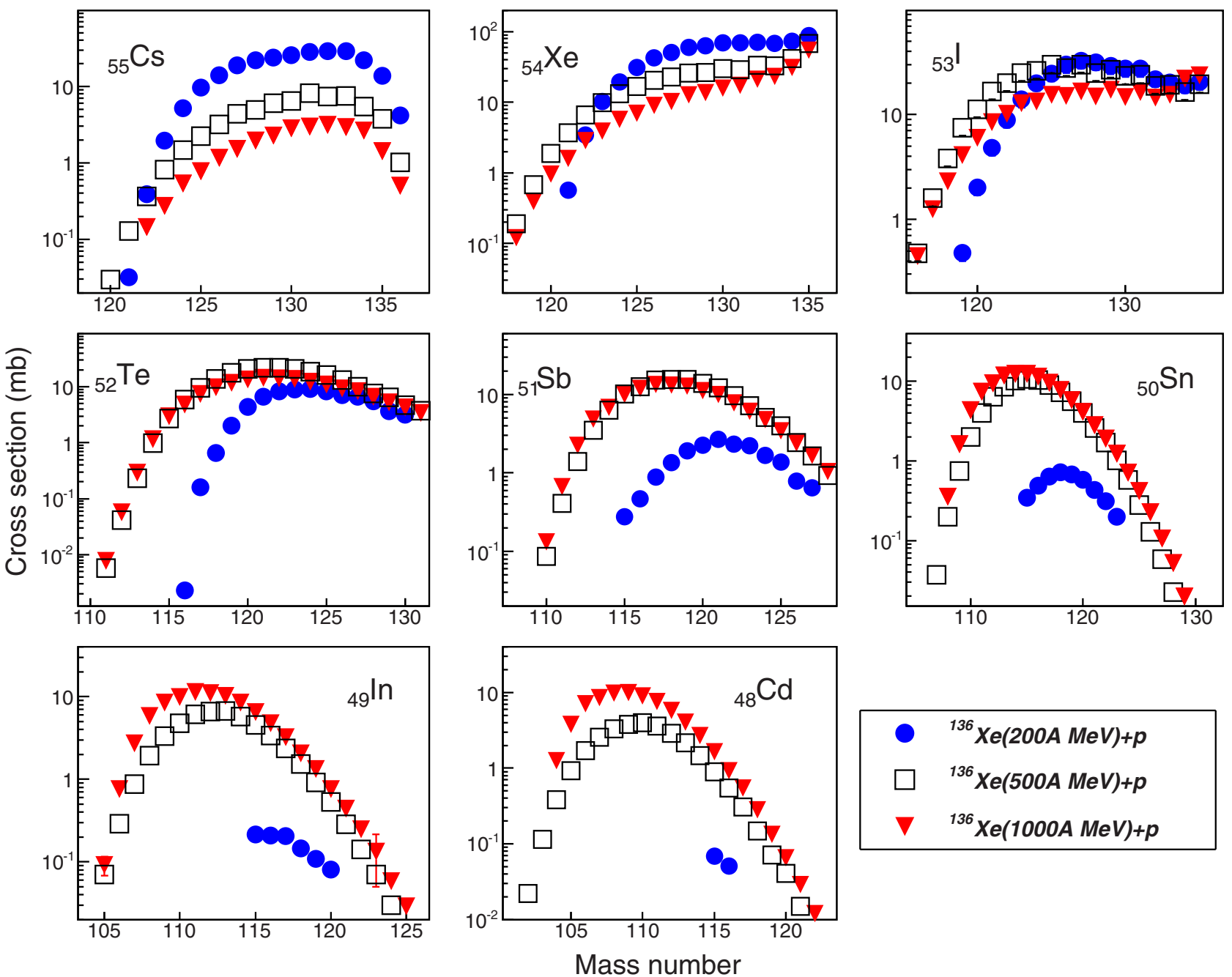

FIG. 15. Isobaric cross sections of the residual nuclei produced in the reactions ${ }^{136} \mathrm{Xe}+p$ at $1000 A \mathrm{MeV}$ (solid triangles) [18], 500A MeV (open squares) [19], and 200 A MeV (solid circles).

cascade $[20,60,61]$ was suggested. During the deexcitation process the prefragments reach the so-called evaporation corridor, where the neutron evaporation competes strongly with other deexcitation channels, such as the emission of light charged particles or intermediate-mass fragments. Therefore, the descent along this corridor characterizes the production of the final evaporation residues and the evolution of their average neutron excess with the excitation energy gained by the prefragments.

However, this pattern is broken by the residual nuclei production for the reaction at $200 \mathrm{~A} \mathrm{MeV}$ (solid circles) in which the lightest residues are much more neutron-rich than at higher energies, as already observed from the isotopic distributions in Fig. 15. This different behavior can be explained by considering that spallation reactions at low energies remove a limited number of nucleons during the intranuclear cascade process and consequently the prefragment excitation energy is not very large. Thus, the prefragments cannot reach the evaporation corridor during the deexcitation process. In that picture we can conclude that at sufficiently high energies the isotopic distributions of the spallation residues are more influenced by the evaporation process while at lower energy they keep memory of the entrance channel, being more directly determined by the cascade process. As can be seen in the Fig. 16, the INCL4.6+ABLA07 calculations describe well this observable for the three energies, indicating that, on average, models describe correctly the energy dissipated during the cascade process, as well as its evacuation by emission of light nucleons and clusters during the deexcitation stage.

In summary, we can conclude that intranuclear-cascade models seem to provide an acceptable description of the excitation energy dissipated in these reactions, provided that the radial distributions of protons and neutrons, and the correlation between kinetic energy and radius of the nucleons inside the nuclear potential are properly described [42]. In fact, the isotopic distributions of residual nuclei not too close in mass number to the initial nucleus and their energy dependence are rather well described by the INCL4.6 model. Surprisingly, these codes present difficulties, at the lower spallation energies, to describe the production of the simplest processes where few nucleons are involved. A clear example is the description of the charge-exchange or nucleon knockout 


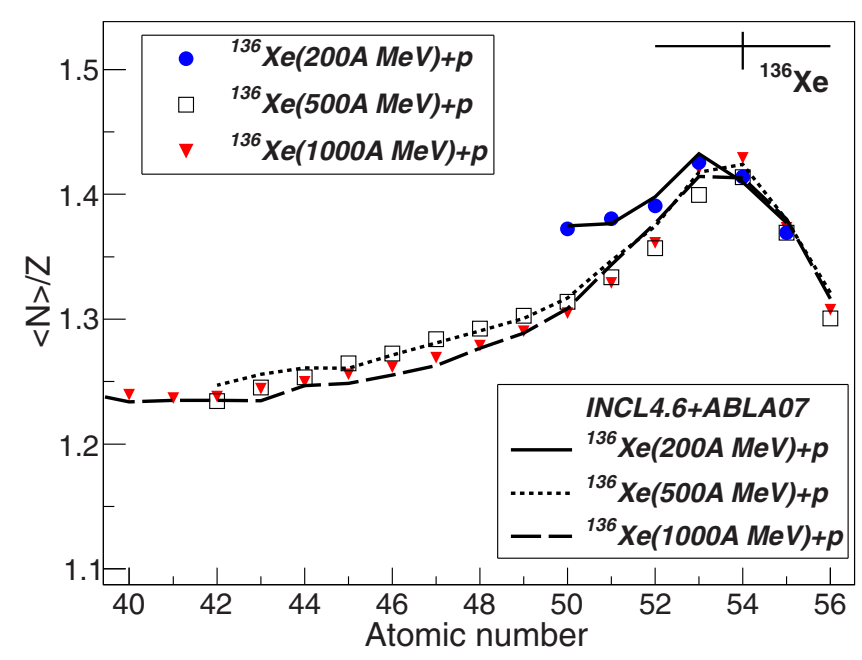

FIG. 16. Average neutron excess of the isotopic distributions of the production cross sections for the residual nuclei measured in the reactions ${ }^{136} \mathrm{Xe}+p$ at $1000 A \mathrm{MeV}$ (triangles), $500 A \mathrm{MeV}$ (squares), and $200 A \mathrm{MeV}$ (solid circles) as a function of the atomic number of the residue. The data are compared with the predictions obtained with INCL4.6 coupled to ABLA07.

channels. These processes should not only be representative of the description of the elementary nucleon-nucleon collisions underlying intranuclear cascade models but they also have major importance because of their large cross sections.

\section{CONCLUSIONS}

In this work, we have presented the first measurement of the isotopic production cross sections of residual nuclei produced in the reaction ${ }^{136} \mathrm{Xe}+p$ at $200 \mathrm{~A} \mathrm{MeV}$. We have obtained the production cross sections of 91 residual nuclei, providing almost complete isotopic distributions from cesium down to tin. The sum of these cross sections together with few extrapolated values provide a total reaction cross section of $1405 \pm 166 \mathrm{mb}$, in agreement with Karol's prediction. The data have been used to benchmarck two intranuclear-cascade models, ISABEL and INCL4.6. In general, the codes reproduce the shape of the isotopic distributions. However, ISABEL underpredicts the production, except for cesium isotopes, and INCL4.6 underpredicts the production of heavy residues.

The results have also been studied together with those obtained for the same reaction at $500 \mathrm{~A}$ and $1000 \mathrm{~A} \mathrm{MeV}$. While the total production cross section remains unchanged with the projectile energy, the isobaric and the isotopic distributions change drastically. This result indicates that the total reaction cross section is mainly determined by the size of the colliding nuclei while the isotopic and isobaric distributions are more sensitive to the energy dissipated. In general, the measured cross sections are compatible with a picture in which the higher incident energies lead to longer nucleon evaporation chains, and consequently, to lighter reaction residues. The comparison of the isotopic distributions at $200 \mathrm{~A}$ and $500 \mathrm{~A} \mathrm{MeV}$ clearly shows a change between a regime where the prefragment gets enough excitation energy in such a way that the final isotopic distribution is determined by an equilibrium between proton and neutron evaporation processes, and a situation where the isotopic composition of the final fragments is mainly determined by the intranuclear-cascade phase.

The puzzling result deduced from this work is that even, if intranuclear-cascade codes provide a reasonable description of the energy dissipated in multiknockout processes, the codes fail in describing the most peripheral reactions involving few nucleon-nucleon collisions. This result may suggest that, already at the lower limit of the spallation regime, mean-field contributions, not considered by INC codes, start having non-negligible effects on the reaction observables. Therefore, additional efforts are in progress to overcome some of the limitations [42].

\section{ACKNOWLEDGMENTS}

This work was financially supported by Sixth Framework Programme through a EURATOM Intra-European Fellowship (SPALLAWASTE Contract, No. 016733). It was partially supported by the Ministerio de Economía y Competitividad under Projects No. FPA2013-47831-C2-1 and No. FPA201569640-C2-1-P, and by the regional Government of Galicia under the program "Grupos de Referencia Competitiva 2013011".
[1] J.-C. David, Eur. Phys. J. A 51, 68 (2015).

[2] J. Benlliure, The Euroschool Lectures on Physics with Exotic Beams, Vol. II (Springer-Verlag, Berlin, 2006).

[3] D. Filges and F. Goldenbaum, Handbook of Spallation Research: Theory, Experiments and Applications (Wiley-VCH, Berlin, 2009).

[4] https://www-nds.iaea.org/spallations.

[5] J. Taieb et al., Nucl. Phys. A 724, 413 (2003).

[6] M. Bernas et al., Nucl. Phys. A 725, 213 (2003).

[7] P. Armbruster, J. Benlliure, M. Bernas, A. Boudard, E. Casarejos, S. Czajkowski, T. Enqvist, S. Leray, P. Napolitani, J. Pereira, F. Rejmund, M.-V. Ricciardi, K.-H. Schmidt, C. Stéphan, J.
Taieb, L. Tassan-Got, and C. Volant, Phys. Rev. Lett. 93, 212701 (2004).

[8] M. V. Ricciardi, P. Armbruster, J. Benlliure, M. Bernas, A. Boudard, S. Czajkowski, T. Enqvist, A. Kelić, S. Leray, R. Legrain, B. Mustapha, J. Pereira, F. Rejmund, K.-H. Schmidt, C. Stéphan, L. Tassan-Got, C. Volant, and O. Yordanov, Phys. Rev. C 73, 014607 (2006).

[9] E. Casarejos, J. Benlliure, J. Pereira, P. Armbruster, M. Bernas, A. Boudard, S. Czajkowski, T. Enqvist, R. Legrain, S. Leray, B. Mustapha, M. Pravikoff, F. Rejmund, K.-H. Schmidt, C. Stéphan, J. Taieb, L. Tassan-Got, C. Volant, and W. Wlazlo, Phys. Rev. C 74, 044612 (2006). 
[10] J. Pereira, P. Armbruster, J. Benlliure, and K.-H. Schmidt, Phys. Rev. C 75, 044604 (2007).

[11] T. Enqvist et al., Nucl. Phys. A 686, 481 (2001).

[12] T. Enqvist et al., Nucl. Phys. A 703, 435 (2002).

[13] B. Fernández-Domínguez et al., Nucl. Phys. A 747, 227 (2005).

[14] L. Audouin et al., Nucl. Phys. A 768, 1 (2006).

[15] F. Rejmund et al., Nucl. Phys. A 683, 540 (2001).

[16] J. Benlliure et al., Nucl. Phys. A 683, 513 (2001).

[17] J. Benlliure et al., Nucl. Phys. A 700, 469 (2002).

[18] P. Napolitani, K. H. Schmidt, L. Tassan-Got, P. Armbruster, T. Enqvist, A. Heinz, V. Henzl, D. Henzlova, A. Kelic, R. Pleskac, M. V. Ricciardi, C. Schmitt, O. Yordanov, L. Audouin, M. Bernas, A. Lafriaskh, F. Rejmund, C. Stephan, J. Benlliure, E. Casarejos, M. FernandezOrdonez, J. Pereira, A. Boudard, B. Fernandez, S. Leray, C. Villagrasa, and C. Volant, Phys. Rev. C 76, 064609 (2007).

[19] L. Giot et al., Nucl. Phys. A 899, 116 (2013).

[20] J. Alcantara-Nuñez et al., Phys. Rev. C 92, 024607 (2015).

[21] P. Napolitani, K.-H. Schmidt, A. S. Botvina, F. Rejmund, L. Tassan-Got, and C. Villagrasa, Phys. Rev. C 70, 054607 (2004).

[22] C. Villagrasa-Canton et al., Phys. Rev. C 75, 044603 (2007).

[23] P. Napolitani and M. Colonna, Phys. Rev. C 92, 034607 (2015).

[24] A. Boudard, J. Cugnon, S. Leray, and C. Volant, Phys. Rev. C 66, 044615 (2002).

[25] C. Schmitt, K.-H. Schmidt, and A. Kelic-Heil, Phys. Rev. C 90, 064605 (2014)

[26] J.-C. David et al., Eur. Phys. J. A 49, 29 (2013).

[27] A. Kelić, K.-H. Schmidt, T. Enqvist, A. Boudard, P. Armbruster, J. Benlliure, M. Bernas, S. Czajkowski, R. Legrain, S. Leray, B. Mustapha, M. Pravikoff, F. Rejmund, C. Stéphan, J. Taïeb, L. Tassan-Got, C. Volant, and W. Wlazło, Phys. Rev. C 70, 064608 (2004).

[28] A. I. Morales, J. Benlliure, J. Agramunt, A. Algora, N. Alkhomashi, H. Alvarez-Pol, P. Boutachkov, A. M. Bruce, L. S. Caceres, E. Casarejos, A. M. DenisBacelar, P. Doornenbal, D. Dragosavac, G. Farrelly, A. Gadea, W. Gelletly, J. Gerl, M. Gorska, J. Grebosz, I. Kojouharov, F. Molina, D. Perez-Loureiro, S. Pietri, Z. Podolyak, P. H. Regan, B. Rubio, H. Shaffner, S. J. Steer, S. Tashenov, S. Verma, and H. J. Wollersheim, Phys. Rev. C 84, 011601 (2011).

[29] I. Leya et al., Nucl. Instrum. Methods Phys. Res., Sect. B 229, 1 (2005).

[30] H. Wang et al., Phys. Lett. B 754, 104 (2016).

[31] H. Geissel et al., Nucl. Instrum. Methods Phys., Res. Sect. B 70, 286 (1992).

[32] A. R Junghans et al., Nucl. Instrum. Methods Phys. Res., Sect. A 370, 312 (1996).

[33] B. Voss et al., Nucl. Instrum. Methods Phys. Res., Sect. A 364, 150 (1995).

[34] M. Pfützner et al., Nucl. Instrum. Methods Phys. Res., Sect. B 86, 213 (1994).

[35] D. Perez-Loureiro et al., Phys. Lett. B 703, 552 (2011).

[36] H. Stelzer et al., Nucl. Instrum. Methods Phys. Res., Sect. A 310, 103 (1991).

[37] T. Kurtukian-Nieto, J. Benlliure, K.-H. Schmidt, L. Audouin, F. Becker, B. Blank, E. Casarejos, F. Farget, M. Fernández-
Ordóñez, J. Giovinazzo, D. Henzlova, B. Jurado, J. Pereira, and O. Yordanov, Phys. Rev. C 89, 024616 (2014).

[38] C. Scheidenberger et al., Nucl. Instrum. Methods Phys. Res., Sect. B 142, 441 (1998).

[39] J. Benlliure, J. Pereira-Conca, and K.-H. Schmidt, Nucl. Instrum. Methods Phys. Res., Sect. A 478, 493 (2002).

[40] K. Sümmerer and B. Blank, Nucl. Phys. A 701, 161 (2002).

[41] P. J. Karol, Phys. Rev. C 11, 1203 (1975).

[42] D. Mancusi, A. Boudard, J. Carbonell, J. Cugnon, J.-C. David, and S. Leray, Phys. Rev. C 91, 034602 (2015).

[43] R. Serber, Phys. Rev. 72, 1114 (1947).

[44] Y. Yariv and Z. Fraenkel, Phys. Rev. C 20, 2227 (1979).

[45] Y. Yariv and Z. Fraenkel, Phys. Rev. C 24, 488 (1981).

[46] A. Boudard, J. Cugnon, J.-C. David, S. Leray, and D. Mancusi, Phys. Rev. C 87, 014606 (2013).

[47] A. Kelic, M. V. Ricciardi, and K.-H. Schmidt, in Proceedings of the Joint ICTP-IAEA Advanced Workshop on Model Codes for Spallation Reactions, ICTP Trieste, Italy, 4-8 February 2008, edited by D. Filges, S. Leray, Y. Yariv, A. Mengoni, A. Stanculescu, and G. Mank, INDC(NDS)-0530 (IAEA, Vienna, 2008), pp. 181-221.

[48] V. F. Weisskopf and D. H. Ewing, Phys. Rev. 57, 472 (1940).

[49] G. Audi, M. Wang, A. H. Wapstra, F. G. Kondev, M. MacCormick, X. Xu, and B. Pfeiffer, Chin. Phys. C 36, 1287 (2012).

[50] R. Bass, in Proceedings of the Symposium on Deep-Inelastic and Fusion Reactions with Heavy Ions, Berlin, 1979 (Springer Verlag, Berlin, 1980).

[51] B. Jurado, K.-H. Schmidt, and J. Benlliure, Phys. Lett. B 553, 186 (2003)

[52] B. Jurado, C. Schmitt, K.-H. Schmidt, J. Benlliure, and A. R. Junghans, Nucl. Phys. A 747, 14 (2005).

[53] B. Jurado, C. Schmitt, K.-H. Schmidt, J. Benlliure, T. Enqvist, A. R. Junghans, A. Kelić, and F. Rejmund, Phys. Rev. Lett. 93, 072501 (2004).

[54] J. Benlliure, E. Casarejos, J. Pereira, and K.-H. Schmidt, Phys. Rev. C 74, 014609 (2006).

[55] C. Schmitt, K.-H. Schmidt, A. Kelić, A. Heinz, B. Jurado, and P. N. Nadtochy, Phys. Rev. C 81, 064602 (2010).

[56] Y. Ayyad, J. Benlliure, E. Casarejos, H. Álvarez-Pol, A. Bacquias, A. Boudard, M. Caamaño, T. Enqvist, V. Föhr, A. Kelić-Heil, K. Kezzar, S. Leray, D. Mancusi, C. Paradela, D. Pérez-Loureiro, R. Pleskač, J. L. Rodríguez-Sánchez, and D. Tarrío, Phys. Rev. C 89, 054610 (2014).

[57] Y. Ayyad, J. Benlliure, J. L. Rodríguez-Sánchez, A. Bacquias, A. Boudard, E. Casarejos, T. Enqvist, M. Fernandez, V. Henzl, V. Henzlova, B. Jurado, A. Kelić-Heil, T. Kurtukian, S. Lukić, P. Nadtochy, D. Pérez-Loureiro, R. Pleskač, F. Farget, M. V. Ricciardi, K.-H. Schmidt, C. Schmitt, and S. N. Ngoc, Phys. Rev. C 91, 034601 (2015).

[58] J. L. Rodríguez-Sánchez et al., Phys. Rev. C 90, 064606 (2014); 91, 064616 (2015); 92, 044612 (2015); 94, 034605 (2016).

[59] J. Cugnon et al., Eur. Phys. J. Plus 131, 169 (2016).

[60] J. P. Dufour et al., Nucl. Phys. A 387, 157 (1982).

[61] R. J. Charity, Phys. Rev. C 58, 1073 (1998). 